\title{
Reverse engineering approach for improving the quality of mobile applications
}

\author{
Eman K. Elsayed ${ }^{1}$, Kamal A. ElDahshan ${ }^{2}$, Enas E. El-Sharawy ${ }^{1}$, Naglaa E. Ghannam ${ }^{\text {Corresp. } 1}$ \\ 1 Department of Mathematical and Computer Science, Faculty of Science, Al Azhar University, (Girls branch), Cairo, Egypt \\ 2 Department of Mathematical and Computer Science, Faculty of Science, Al Azhar University, Cairo, Egypt \\ Corresponding Author: Naglaa E. Ghannam \\ Email address: naglaasaeed@azhar.edu.eg
}

Background: Portable-devices applications (Android applications) are becoming complex software systems that must be developed quickly and evolve continuously to fit new user requirements and execution contexts. Applications must be produced rapidly and advance persistently in order to fit new client requirements and execution settings. However, catering to these imperatives may bring about poor outline decisions on design choices, known as anti-patterns, which may possibly corrupt programming quality and execution. Thus, the automatic detection of anti-patterns is a vital process that facilitates both maintenance and evolution tasks. Additionally, it guides developers to refactor their applications and consequently enhance their quality.

Methods: We proposed a general method to detect mobile applications' anti-patterns that can detect both semantic and structural design anti-patterns. The proposed method is via reverse-engineering and Ontology by using UML modeling environment, OWL ontology-based platform and Ontology-driven conceptual modeling. We present and test a new method that generates OWL Ontology of mobile applications and analyzes the relationships among object-oriented anti-patterns and offer methods to resolve the anti-patterns by detecting and treating 15 different design's semantic and structural antipatterns that occurred in analyzing of 29 mobile applications. We choose 29 mobile applications randomly. Selecting a browser is not a criterion in this method, because the proposed method is applied on a design level. We demonstrate a semantic integration method to reduce the incidence of antipatterns using the Ontology merging on mobile applications.

Results: The proposed method detected 15 semantic and structural design anti-patterns which have appeared 1262 times in a random sample of 29 mobile applications. The proposed method introduced a new classification of the anti-patterns divided into four groups. "The anti-patterns in the class group" is the most group that has the maximum occurrences of anti-patterns and "The anti-patterns in the operation group" is the smallest one that has the minimum occurrences of the anti-patterns which are detected by the proposed method. The results also showed the correlation between the selected tools which we used as Modelio, Protégé platform and OLED editor of the OntoUML. The results showed that there was a high positive relation between Modelio and Protégé which implies that the combination between both increases the accuracy level of the detection of anti-patterns. In the evaluation and analyzing the suitable integration method, we applied the different methods on homogeneous mobile applications and found that using Ontology increased the detection percentage approximately by $11.3 \%$ in addition to guaranteed consistency. 
1 Reverse engineering approach for improving the quality of 2 mobile applications

3

4

5

6

7

8

9

10

11

12

13

14

15

16

17

18

19

20

21

22

23

24

25

26

27

28

29

30

31

32

33

34

35

36

37

38

39

40

\section{Eman K. Elsayed ${ }^{1}$, Kamal A. ElDahshan ${ }^{2}$, Enas E. El-Sharawy ${ }^{1}$, Naglaa E. Ghannam ${ }^{1}$}

${ }^{1}$ Department of Mathematical and Computer Science, Faculty of Science, Al-Azhar University (Girls branch), Cairo, Egypt.

${ }^{2}$ Department of Mathematical and Computer Science, Faculty of Science, Al-Azhar University, Cairo, Egypt.

Corresponding Author:

Naglaa E. Ghannam

Cairo, Egypt

Email address: naglaasaeed@azhar.edu.eg

\section{Abstract}

Background: Portable-devices applications (Android applications) are becoming complex software systems that must be developed quickly and evolve continuously to fit new user requirements and execution contexts. Applications must be produced rapidly and advance persistently in order to fit new client requirements and execution settings. However, catering to these imperatives may bring about poor outline decisions on design choices, known as antipatterns, which may possibly corrupt programming quality and execution. Thus, the automatic detection of anti-patterns is a vital process that facilitates both maintenance and evolution tasks. Additionally, it guides developers to refactor their applications and consequently enhance their quality.

Methods: We proposed a general method to detect mobile applications' anti-patterns that can detect both semantic and structural design anti-patterns. The proposed method is via reverse-engineering and Ontology by using UML modeling environment, OWL ontology-based platform and Ontology-driven conceptual modeling. We present and test a new method that generates OWL Ontology of mobile applications and analyzes the relationships among object-oriented anti-patterns and offer methods to resolve the anti-patterns by detecting and treating 15 different design's semantic and structural anti-patterns that occurred in analyzing of 29 mobile applications. We choose 29 mobile applications randomly. Selecting a browser is not a criterion in this method, because the proposed method is applied on the design level. We demonstrate a semantic integration method to reduce the incidence of anti-patterns using the Ontology merging on mobile applications.

Results: The proposed method detected 15 semantic and structural design anti-patterns which have appeared 1262 times in a random sample of 29 mobile applications. The proposed method introduced a new classification of the anti-patterns divided into four groups. "The anti-patterns in the class group" is the most group that has the maximum occurrences of anti-patterns and "The anti-patterns in the operation group" is the smallest one that has the minimum occurrences of the anti-patterns which are detected by the proposed method. The results also showed the correlation between the selected tools which we used as Modelio, Protégé platform and OLED editor of the OntoUML. The results showed that there was a high

Peer] Comput. Sci. reviewing PDF | (CS-2019:03:35864:1:2:NEW 11 Jun 2019) 
41

42

43

44

45

46

47

48

49

50

51

52

53

54

55

56

57

58

59

60

61

62

63

64

65

66

67

68

69

70

71

72

73

74

75

76

77

78

79

80

81

positive relation between Modelio and Protégé which implies that the combination between both increases the accuracy level of the detection of anti-patterns. In the evaluation and analyzing the suitable integration method, we applied the different methods on homogeneous mobile applications and found that using Ontology increased the detection percentage approximately by $11.3 \%$ in addition to guaranteed consistency.

\section{Introduction}

Mobile applications take center stage in our lives today. We utilize them anywhere, at any time and for everything. We use them to peruse websites, shop, search for everything we need and for basic administration such as banking. For the importance of mobile applications, their reliability and quality are critical. Like any other applications, the initial design of mobile applications is affected by bug-settling and the introduction of new properties, which change the initial design; this can occasionally affect the quality of design (Parnas D. L, 1994). This aspect is known as software degeneration, which can exist in the form of design flaws or anti-patterns (Eick S. G. et al. 2001).

One of the most important factors in the development of software systems is improving software quality. The success of software design depends on the availability of quality elements such as maintainability, manageability, testability, and performance. These elements are adversely affected by anti-patterns (Afjehei, S. S. et al. 2019, Yamashita, A., \& Moonen, L. 2013). Antipatterns are bad practice in software design. The automatic detection of anti-patterns is a good way to support maintenance, uncomplicate evolution tasks and improve usability. In addition to the general advantages of detecting anti-patterns, we think that detecting anti-patterns provides developers with a way to ensure that the detected anti-patterns will not be repeated in applications revisions. Also, detecting anti-patterns may improve both operational characteristics and user experience.

We noted that many other approaches interested in detecting anti-patterns in the code level as introduced by Morales et al., (2016) and Alharbi et al. (2014). Although, it has been noted that anti-pattern detection at the design level reduces many code anti-patterns and is more general.

According to Raja, V. (2008), engineering is the process of designing, manufacturing, assembling, and maintaining products and systems. Engineering has two types, forward engineering, and reverse engineering as presented by Raja, V. (2008). Chikofsky, E. J., \& Cross, J. H. (1990) defined reverse engineering (RE) as the process of analyzing software systems to identify the components of the systems and the interrelationships between them and presenting the systems in other forms or at a higher level of abstraction. The term Reverse Engineering according to our approach, refers to the process of generating UML diagrams followed by generating OWL ontologies of mobile applications through importing and analyzing the bytecode.

Generally, we can use Ontology re-engineering for direct incorporation as an ontology development method (Obrst et al., 2014) by allowing the designer to analyze the common components dependence.

Designing a pattern of mobile application remains an ongoing research challenge. The proposed approach aims to detect structural and semantic anti-patterns in the design of mobile applications. Also, to show which method is better for the integration of applications. 
82 Motivated by the research mentioned above, the major contributions of this paper are six-fold:

83 - Presenting a new method for generating OWL Ontology of mobile applications.

84 - Presenting a general method for enhancing the design of a pattern of a mobile application.

85 - Illustrating how the proposed method can detect both structural and semantic anti-patterns in the 86 design of mobile applications.

87 - Describing how we evaluate the proposed method in 29 mobile applications. Showing how it 88 detects and treats 15 designs' semantic and structural anti-patterns that appeared 1262 times.

89 - Showing how semantic integration among mobile applications decreases the occurrences of anti90 patterns in the generated mobile application pattern.

91 - Analyzing the relationships among the object-oriented anti-patterns and the detection tools.

92 In the rest of the paper, we subsequently present the related work. Next, we present some basic 93 definitions, and the details of the proposed approach is described. After that, the empirical 94 validations of the proposed method are presented, followed by the results and discussion. And, 95 finally, the concluding remarks are given, along with scope for future work.

96

97 Related Works

98 Many empirical studies have demonstrated the negative impact of anti-patterns on change99 proneness, fault-proneness, and energy efficiency (Romano et al., 2012; Khomh et al., 2012; 100 Morales et al., 2016). In addition to that, Hecht et al. (2015); Chatzigeorgiou \& Manakos (2010); Hecht et al. (2016) observed an improvement in the user interface and memory performance of mobile apps when correcting Android anti-patterns. They found that anti-patterns were prevalent in the evolution of mobile applications. They also confirmed that anti-patterns tend to remain in systems through several releases unless a major change is performed on the system. Many efficient approaches have been proposed in the literature to detect mobile applications' anti-patterns. Some researchers concentrate on ensuring that the soft is free of contradictions which are called consistency. Alharbi et al. (2014) detected the anti-patterns related to inconsistency in mobile applications that were only related to camera permissions and similarities. Joorabch et al. (2015) detected the anti-patterns related to inconsistency in mobile applications using a tool called CHECKCAMP that was able to detect 32 anti-patterns related to inconsistencies between application versions. Hecht et al. (2015) used the Paprika approach to detect some popular objectoriented anti-patterns in the code of mobile applications using threshold technique. LinaresVásquez et al. (2014) detected 18 Object Oriented (OO) anti-patterns in 1,343 java mobile 114 applications by using DÉCOR. This study focused on the relationship between smell anti-patterns and application domain. Also, they showed that the presence of anti-patterns negatively impacts software quality metrics, in particular, metrics related to fault-proneness. Yus, R., \& Pappachan, P. (2015) analyzed more than 400 semantic Web papers, and they found that more than 36 mobile applications are semantic mobile applications. They showed that the existence of semantic helps in better local storage and battery consumption. The detection of semantic anti-patterns will improve the quality of mobile applications. Palomba et al. (2017) proposed an automated tool called A DOCTOR. This tool can identify 15 Android code smells. They made an empirical study 
122 conducted on the source code of 18 Android applications and revealed that the proposed tool

123

124

125

126

127

128

129

130

131

132

133

134

135

136

137

138

139

140

141

142

143

144

145

146

147

148

149

150

151

152

153

154

155

156

157

158

159

160

161

reached $98 \%$ precision and $98 \%$ recall. A DOCTOR detected almost all the code smell instances existing in Android applications. Hecht et al. (2015) introduced the PAPRIKA tool to monitor the evolution of mobile application quality based on anti-patterns. They detected the common antipatterns in the code of the analyzed applications. They detected seven anti-patterns. Three of them were Object Oriented anti-patterns and four are mobile applications anti-patterns.

Reverse engineering (RE) is the process of analyzing software systems to identify the components of the systems and the interrelationships between them and presenting the systems in other forms or at a higher level of abstraction Chikofsky, E. J., \& Cross, J. H. (1990).

In this paper, we used reverse engineering to transfer code level to design level for detecting mobile applications' anti-patterns. RE techniques are important for understanding the construction of the user interface and algorithms of applications. Additionally, we can know all the properties of the application, its activities, and permissions and can read the Mainfest.xml of the applications. RE techniques have been used with mobile applications for many purposes not just for detecting antipatterns. Song, L. et al. (2017) used RE for improving the security of Android applications. While Zhou, X. et al. (2018) used the RE technique to detect logging classes and to remove logging calls and unnecessary instructions. Also, Arnatovich, Y. L et al. (2018) used RE to perform program analysis on a textual form of the executable source and to represent it with an intermediate language (IL). This IL has been introduced to represent applications executable Dalvik (dex) bytecode in a human-readable form.

\section{Ontology and Software Engineering}

According to the IEEE Standard Glossary (1990), software engineering is defined as "the application of a systematic, disciplined, quantifiable approach to the development, operation, and maintenance of software".

Also, from the knowledge engineering community perspective, computational ontology is defined as "explicit specifications of a conceptualization". According to Calero et al. (2006); Happel. J., \& Seedorf, S. (2006), the importance of sharing knowledge to move the software to more advanced levels require an explicit definition to help machines interpret this knowledge. Happel. J., \& Seedorf, S. (2006) decided that ontology is the most promising way to address software engineering problems.

El-Sayed et al., 2016 proofed the similarities in infrastructures between UML and ontology components. They proposed checking some UML quality features using ontology and ontology reasoning services to check consistency and redundancies over UML models. This would lead to a strong relationship between software design and ontology development.

In software engineering, ontologies have a wide range of applications, including model transformations, cloud security engineering, decision support, search and semantic integration (Kappel et al., 2006; Aljawarneh et al., 2017; Maurice et al., 2017; Bartussek et al., 2018; De Giacomo et al. 2018). Semantic integration is the process of merging the semantic contents of multiple ontologies. The integration may be between applications that have the same domain or

Peer] Comput. Sci. reviewing PDF | (CS-2019:03:35864:1:2:NEW 11 Jun 2019) 
162 have different domains to take the properties of both applications. We make ontology integration

163 for many reasons: to reuse the existing semantic content of applications, to reduce effort and cost,

164 to improve the quality of the source content or the content itself, and to fulfill user requirements

165 that the original ontology does not satisfy.

166

167

168

169

170

171

172

\section{Proposed Method}

In this section, we introduce the key components of the proposed method for analyzing the design of mobile applications to detect design anti-patterns, and for making semantic integration between mobile applications via ontology reengineering.

173

The proposed method for anti-pattern detection consists of three main phases and is summarized in Fig. 1. Also, there is an optional phase called the integration phase.

1. The first phase presents the process of reformatting the mobile application to Java format.

175

2. The second phase presents the reverse-engineering process. In this phase, we used RE to reverse the Java code of mobile applications and generating UML class diagram models. Additionally, a lot of design anti-patterns are detected. The presented reverse approach is accurate enough to analysis the information that we need about APK to reverse UML models of the applications. converts UML mobile application model to OWL Ontology, then analyzes the relationships among object-oriented anti-patterns and offers methods to resolve the anti-patterns related to semantic and inconsistency. After that, we can regenerate the Java code of mobile applications. The developer can ensure that anti-patterns in existing applications will not be repeated in application revisions and may improve both operational characteristics and user experience. by merging the OWL ontologies of both applications. From these two ontologies, it will be yield one integrated application for doing both services with minimum anti-patterns. We will present in details the rationale provided for why this integration is needed as an optional phase if we need.

\section{The Integration of Mobile Applications}

190

191

192

193

194

195

196

197

198

199

200
The integration process is most for the inclusion of new skill sets for applications such as IOT or monitoring applications or potentially voice-activation integration into an existing application. But, here we interested in presenting a new manner for homogenous integration to combine the advantages of two mobile applications in a new pattern. In this section, we provided a rationale for why this integration is needed and presenting the integration as an extra phase if we need. Where the other detection phases don't change. Patterns are advanced methods to develop any mobile applications. The integration or merging of mobile applications is a good step in mobile application development. The advantage of the integration of mobile applications is responding to the puzzling selection of the appropriate application from a set of applications. This will achieve the same objective if each application has a different advantage and the developer wants to start to improve pattern combines all advantage without anti-patterns. 
201

202

203

204

205

206

207

208

209

210

211

212

213

214

215

216

217

218

219

220

221

222

223

224

225

226

227

228

229

230

231

232

233

234

235

236

237

238

239

240

241

242

243

To clear our idea, we choose two homogenous applications as Viber and WhatsApp. They are the most popular messaging and VoIP (Voice Over IP) applications. Both Viber and WhatsApp are similar in services, features, security, and costs. Where, both are plenty to like, produce the same services as end-to-end encryption, support groups and video calls, support on any operating system, allow transmission of documents or multimedia, and work over $3 \mathrm{G}, 4 \mathrm{G}$ and Wi-Fi. Well, both are fantastic in their way. But, to know which one is better for the developer to be a pattern for his refinement? We found that Viber has been offering both video and voice calling for a far longer time than WhatsApp and has hidden chat feature. Also, Viber lets you play a list of games you can play along with other Viber contacts. But, WhatsApp is popular and easy to use. We can make the integration of both applications and take the best skills of both.

We imagine that when producing a new application we can directly integrate it to the old one without replacing.

In the case of heterogonous integration applications, the developer, for example, may want to develop a new health care hybrid application. From website "free apps for me" https://freeappsforme.com/ developer can find at least 7 applications for measuring blood pressure. All of them are free and available on a different platform. There are also at least 13 Diabetes applications. When developer merging two applications one for measuring blood pressure as "Smart Blood Pressure" and the other for controlling diabetes as "OneTouch Reveal application, the integration phase will yield one integrated application for doing both services. That is with minimum anti-patterns. Then the developer can add the new relations between these diseases controller without conflict.

The integration allows the combination of the skills of both applications to get new mobile application pattern. These two examples of two types of integration answer the question of why we need to integrate mobile applications.

We suggest using the integration pattern, then comparing between the two integration proposed methods to select the suitable one.

The first integration method is after decompiling the APK of the applications. We use reverse engineering methodology for generating one UML class diagram of both applications. Then we starts the detection of the anti-patterns process for the integrated application (Fig. 2).

The second integration method is through merging the OWL ontologies of both applications using Prompt the plugin in protégé the editor of Ontology as introduced in (Fig. 3).

\section{The Implementation}

In this section, we propose the implementation of the proposed detection method and determine which packages are suitable for each phase.

- The First Phase: APK files are zip files used for the installation of mobile apps. We used the unzip utility for extracting the files stored inside the APK. It contained the AndroidManifest.xml, classes.dex containing the Java classes we used in the reverse process, and resources.arsc containing the meta-information. We de-compiled the APK files using apktool or Android decompiler. Android de-compiler is a script that combines different tools to successfully de-compile any (APK) to its java source code and resources. Finally, we used a java de-compiler tool such as JD-GUI to de-compile the java classes. JD-GUI is a standalone graphical utility that displays the Java code of ".class" files. The input of the first phase was the APK file of the mobile application 
244 and the output was the java classes of the APK application. JD-GUI is accurately enough to

245

246

247

248

249

250

251

252

253

254

255

256

257

258

259

260

261

262

263

264

265

266

267

268

269

270

271

272

273

274

275

276

277

278

279

280

281

282

283 generate the java code that we use to reverse the models of the applications.

- The Second Phase: We used a RE approach for generating the UML class diagram models of the mobile applications. (Elsayed, E., et al. (2019)) compared between UML tools, the authors found that Modelio 3.6 is a suitable tool for modeling and detecting UML design anti-patterns. The UML class diagram was generated by reversing the java binaries of the mobile app. Detecting anti-patterns in the UML model is the first step in the detection process. The input of the second phase was classes.java of the app and the output was the UML class diagram model of the app with a list of the detected anti-patterns.

- The Third Phase: By converting the model to XML format, we could generate it as an OntoUML model in OLED, which is the editor of OntoUML for detecting semantic anti-patterns. OntoUML is a pattern-based and ontologically well-founded version of UML. Its meta-model has been designed in compliance with the ontological distinctions of a well-grounded theory named the Unified Foundational Ontology (UFO). OLED editor also supports the transformation of the OLED file to the OWL ontology of the mobile app, allowing the detection of inconsistency and semantic anti-patterns using the 'reasoner' ontology in Protégé. Protégé is the broad ontology editor commonly used by many users.

The integration phase (the forth optional phase): we propose two methods for integrating mobile applications. The first method is merging the UML models at the second phase when we reverse the models from java code and then completing the detection phases over the integrated application. The second method is merging the OWL Ontologies of the both applications using a Prompt (Protégé plugin) to generate one OWL Ontology pattern. Figure 4 shows the both applications "Viber and WhatsApp" components before merging. Figure 5 shows the integrated application; Fig. 5 has 3 tabs, (classes, slots, and instances) which are the components of the Ontology. Every tab shows the components of its type after integration. Finally, we used "Reasoner in Protégé" to check the consistency after integration.

\section{Empirical Validations}

We assessed our approach by reporting the results we obtained for the detection of 15 anti-patterns on a random sample of 29 popular Android applications downloaded randomly from the APK Mirror.

\section{Applications under Analysis}

Table 1 presents the downloaded applications from the APK Mirror. We selected some popular applications such as YouTube, WhatsApp, Play Store, and Twitter. The size of the applications included the resources of the application, as well as images and data files (Table 1). The research study included the identification and repetition of anti-patterns across different domains and different sizes.

\section{Case Study on "Avast Android Mobile Security"}

To explain the proposed method, we presented a snapshot of it in a different case study "Avast Android Mobile Security". The case study is one of the 29 mobile applications that is proposed in this article for the evaluation of the proposed method. The case study is downloaded from the 
284 APKMirror. The "Avast Android Mobile Security" secures the devices against phishing attacks 285 from emails, phone calls, infected websites, or SMS messages. Also, it has many other features as 286 Antivirus Engine, App Lock, Call Blocker, Anti-Theft, Photo Vault, VPN (virtual private 287 network), and Power Save. The reason for choosing "the Avast Android Mobile Security" 288 application as a case study is that it has the maximum number of the detected anti-patterns using 289 the proposed method. Using the reverse methodology, we generated the UML class diagram model 290 of the java classes in Modelio. The model includes the classes, subclasses, class attributes, 291 operations, and the associations between them (Fig. 6).

292 After generating the UML class diagram of the application in Modelio, we detected 229 repeated 293 anti-patterns in the 'Avast Android Mobile Security'. The anti-patterns are shown in Fig. 7. The 294 number and the location of the anti-patterns were determined.

295 There were 10 detected anti-patterns (without repeat): "NameSpaces have the same name 296 (NHSN)", "NameSpace is Leaf and is derived (NLAD)", "NameSpace is Leaf and is abstract 297 (NLAA)", "Generalization between two incompatible elements (GBUE)", "A public association 298 between two Classifiers one of them is public and the other is privet (PACPP)", "Classifier has 299 several operations with the same signature (CHSO)", "Classifier has attributes with the same name 300 (CHSA)", "The status of an Attribute is abstract and class(SAAC)", "A destructor has two 301 parameters (ADHPS)" and finally "MultiplicityMin must be inferior to MultiplicityMax 302 (MMITMM)". Figure. 8 shows a sample of them.

303 To convert the UML model to XML format, we converted it into an enterprise architecture file 304 (EA) then converted it to an OLED file. In the "Avast Android Mobile Security' OLED file, we 305 validated the model for detecting the anti-patterns. The detected anti-patterns in the different apps 306 were: Association Cycle anti-patterns (AC), Binary relations with Overlapping Ends anti-patterns 307 (BinOver), Imprecise Abstraction (ImpAbs) anti-patterns and Relation Composition anti-patterns 308 (RC)).

309 After anti-patterns detection using OntoUML editor, OLED supports the transformation of OLED 310 file to the OWL Ontology. We checked the inconsistency anti-patterns using the reasoner of 311 Ontology editor (Protégé). The reasoner detected the anti-patterns related to inconsistency as 312 (Similar name, Multiplicity constraints and Cyclic Inheritance). Using the reasoner of Ontology 313 over the case study, we detected the anti-patterns in the classes that have the anti-patterns 314 NameSpaces have the same name, Classifier has several operations with the same signature, 315 Classifier has attributes with the same name, and MultiplicityMin must be inferior to 316 MultiplicityMax, which we detected after generating the class diagram in Modelio, and detected 317 the anti-pattern (Association Cyclic) which was detected via OLED.

318 The treatment or correction of the detected anti-patterns is classified into the following:

319 - Modelio presents the solution as a list of recommendation which developer can do it manually. 320 In this case study, Table 2 presents the anti-patterns and the method of correction.

321 - OLED presents automatic solutions to correct the anti-patterns which we list in 'Table 3'.

322 - Reasoner in Protégé presents all inconsistency anti-patterns where, Reasoner gives just the 323 location of the inconsistent classes as in (Fig. 9). 


\section{Results and Discussion}

325 We applied our proposed method on a sample of 29 Android applications, which we downloaded

326

327

328

329

330

331

332

333

334

335

336

337

338

339

340

341

342

343

344

345

346

347

348

349

350

351

352

353

354

355

356

357

358

359

360

361

362

363

from the APK Mirror. The results present the detected anti-patterns in the 29 mobile applications and the relation between the different types of anti-patterns. The proposed method detected 15 anti-patterns. The total number of anti-patterns that appeared in the 29 applications was 1262 anti-patterns. We classified the anti-patterns according to their existence in the UML class diagram components. The occurrences of the anti-patterns are given in Table 4. Every group has the anti-patterns that were detected in it. For example, the group "Anti-patterns in Operations" presents all anti-patterns that were detected in the operations using the three tools.

Table 5 shows the detected anti-patterns in each application using the proposed method and the total number of anti-patterns in the 29 mobile applications.

We found that the "Anti-patterns in the class" group is the most commonly detected anti-pattern in Android applications. The "Anti-patterns in Operation" is the least commonly appeared antipattern (Fig. 10).

We measured the relations between anti-patterns groups using correlation coefficient. Correlation coefficient is a statistical measure of the degree to which changing the value of one variable predict changing to the value of the other. A positive correlation indicates that the extent to which those variables increase or decrease in parallel. While a negative correlation indicates the extent to which one variable increases as the other decreases. Table 6 presents the correlations between antipatterns groups. The tool can detect certain group, it also can detect in parallel the other as attributes anti-patterns with operations anti-patterns. Also, appearance of attributes anti-patterns in certain applications indicates the appearance of operations anti-patterns strongly. Then the correlation between the five groups of anti-patterns is used to know if the existence of any type of them implies the existence of other type. There was a strong negative correlation (-.1) between Namespaces anti-patterns and Association anti-patterns. Also, a strong positive correlation (.8) between Attributes anti-patterns and Operations anti-patterns.

Also, we analyzed the correlation between the detection tools of the proposed method (Table 7). The greatest correlations were between Modelio and Protégé. For assessing the direct relation between Protégé and Modelio, we calculated the statistical means of anti-patterns which were detected by each tool (Modelio, Protégé, and OLED) on 29 mobile applications as in Fig 11.Figure 11 shows the similarity between both the means of Protégé and Modelio as the result of the correlation. Now, we want to answer statistically the question "Are we need to use the three tools?". And "is there a relation between them?"

For statistical analysis to explain the relation among the three tools and the anti-patterns' groups, we used the analysis of variance ANOVA test. That is to determine whether there are any statistically significant differences between the means of anti-patterns detection by each one of the tools. Also, if there any relation between anti-patterns groups and the features of mobile applications.

We use ANOVA to calculate a test (F-ratio) with which we can obtain the probability P-value (usually taken as $\mathrm{P}<0.05$ ) suggests that at least one group mean is significantly different from the

Peer) Comput. Sci. reviewing PDF | (CS-2019:03:35864:1:2:NEW 11 Jun 2019) 
364

365

366

367

368

369

370

371

372

373

374

375

376

377

378

379

380

381

382

383

384

385

386

387

388

389

390

391

392

393

394

395

396

397

398

399

400

401

402

403

404

others. The null hypothesis is (all population means are equal). The alternative hypothesis is (at least one population mean is different from the rest). Where the degree of freedom (df) between groups is 28 and df within the group is 116 . We found that the significant differences are 0.578 , 0.464 and 0.926 for Protégé, Modelio, and OLED respectively. This implies that the null hypothesis is false i.e. all the detection tools are necessary and required for the detection of the anti-patterns.

ANOVA proof statistically that there was no concerning in the features or the specifications of the applications. That is from low F-value means the group means are close together relative to the variability within each group.

We separated the result of integration phase because it's an optional phase. In the case of homogeneous applications, we found that the number of the detected anti-patterns in the output application was not the same. The detected anti-patterns using the Ontology Integration tool Prompt was less than the number of anti-patterns detected by using the Modelio tool. This indicates that semantic integration decreases the increases the accuracy of detecting anti-patterns in mobile applications. Table 8 shows the number of anti-patterns in each application in the integration case study (Viber \& WhatsApp) and the number of them in the mobile application pattern after merging. The enhancement using Ontology is approximately by $11.3 \%$ in addition to a consistency check. Where the formula to calculate the increasing percent between two values is

$$
\text { Percent increase }=[(\text { second value }- \text { first value }) / \text { first value }] * 100
$$

Substitute in formula (1) by

The first value is the total number of anti-patterns according to using Modelio $=115$.

The second value is the total number of anti-patterns according to using Prompt $=128$.

Then the percent is increased by $\cong 11.3 \%$ which implies that using Ontology integration by Prompt (Protégé plugin) instead of using UML integration by Modelio increases the percent of detection.

Additionally, using ontology to separately refine Viber or WhatsApp as a pattern enhanced them approximately $4.04 \%$ and $89 \%$, respectively, in addition to a consistency check by "Reasoner".

\section{Conclusions}

In this paper, we focused on improving mobile applications' quality. We introduced a general method to automatically detect anti-patterns not by using specific queries, but by using Modelio, OLED, and Protégé in a specific order to get positive results. Also, concerning the related work section, our proposed method is more general than other methods as the proposed method supports semantic and structural anti-pattern detection at design level.

For evaluation of the proposed method, we applied it on a sample of 29 mobile applications. It detected 15 semantic and structural design anti-patterns. According to the proposed classification of anti-patterns, "the anti-patterns in the class group" was the most frequent anti-pattern, and "the anti-patterns in the attribute group" was the least frequent. From the perspective of anti-patterns detection, the analysis of results also showed that there is a correlation between the Modelio and Protégé platforms. Also, there is no correlation between OLED and Protégé and no correlation between Modelio and OLED.

Peer] Comput. Sci. reviewing PDF | (CS-2019:03:35864:1:2:NEW 11 Jun 2019) 
405 We found that using Ontology in the integration phase increases the detection percentage 406 approximately by $11.3 \%$ and guarantees consistency which is assessed by the reasoner of the 407 Ontology. Accordingly, semantic Ontology integration has a positive effect on the quality of the 408 new application. It helps developing a correct, consistent and coherent integrated pattern that has 409 few anti-patterns.

410 Finally, we recommend the developer before using any mobile application as a pattern, helshe 411 should check the design of the selected application against the anti-patterns.

412 When developer concerned with avoiding certain anti-patterns type, the correlations between anti413 patterns groups and between tools will help him. Also, the proposed method considered the issues 414 and problems of developers who are revising Android applications and integrating new packages 415 of code skill sets. A code review such as the methodology proposed could be very valuable in 416 terms of not carrying forward existing anti-patterns and not incorporating new code flawed with 417 poor design. The reverse deeply in OWL ontology of a mobile application very useful.

418 In the future, we are going to solve the problem of big ontologies which cannot be opened in 419 Ontology editors as Protégé to complete the detection process. Although, detection of anti-patterns 420 at design level is very useful and reduces some anti-patterns in the code level, but we will refine 421 the metric method for detecting code level anti-patterns on big Ontology. Also, we will create a 422 semantic web application for anti-patterns to collect all detection tools of the two levels and anti423 patterns catalog. Finally, the correction phase in Modelio and Reasoner are still open issue.

\section{References}

425 Afjehei, S. S., Chen, T. H. P., \& Tsantalis, N. (2019). iPerfDetector: Characterizing and 426 detecting performance anti-patterns in iOS applications. Empirical Software Engineering, 1-30. 427 Alharbi, K., Blackshear, S., Kowalczyk, E., Memon, A. M., Chang, B. Y. E., \& Yeh, T. (2014, 428 April). Android apps consistency scrutinized. In CHI'14 Extended Abstracts on Human Factors 429 in Computing Systems, 26 April - 01 May 2014; Toronto, Ontario, Canada. New York, NY, 430 USA: ACM. pp. 2347-2352. ACM.

431 Aljawarneh, S. A., Alawneh, A., \& Jaradat, R. (2017). Cloud security engineering. Future 432 Generation Computer Systems, 74(C), 385-392.

433 Arnatovich, Y. L., Wang, L., Ngo, N. M., \& Soh, C. (2018). A Comparison of Android Reverse 434 Engineering Tools via Program Behaviors Validation Based on Intermediate Languages

435 Transformation. IEEE Access, 6, 12382-12394.

436 Bartussek, W., Weiland, T., Meese, S., Schurr, M. O., Leenen, M., Uciteli, A., ... \& Lauer, W. 437 (2018, April). Ontology-based search for risk-relevant PMS data. In Biomedical Engineering 438 Conference (SAIBMEC), 1-4. IEEE.

439 Calero, C., Ruiz, F., \& Piattini, M. (Eds.). (2006). Ontologies for software engineering and 440 software technology. Springer Science \& Business Media.

441 Chatzigeorgiou, A., \& Manakos, A. (2010, September). Investigating the evolution of bad smells 442 in object-oriented code. In Seventh International Conference on the Quality of Information and 443 Communications Technology (QUATIC), 10, 106-115. IEEE. 
444 Chikofsky, E. J., \& Cross, J. H. (1990). Reverse engineering and design recovery: A taxonomy. 445 IEEE software, 7(1), 13-17.

446 De Giacomo, G., Lembo, D., Lenzerini, M., Poggi, A., \& Rosati, R. (2018). Using ontologies for 447 semantic data integration. In A Comprehensive Guide Through the Italian Database Research 448 Over the Last 25 Years, 187-202. Springer, Cham.

449 Eick, S. G., Graves, T. L., Karr, A. F., Marron, J. S., \& Mockus, A. (2001). Does code decay? 450 assessing the evidence from change management data. IEEE Transactions on Software

451 Engineering, 27(1), pp. 1-12.

452 Elsayed, E., El-Dahshan, K., El-Sharawy, E., \& Ghannam, N. (2016). Semantic Anti-patterns

453 Detection in UML Models based on Ontology Catalogue. Artificial Intelligence and Machine 454 Learning Journal, 16, 1687-4846.

455 Elsayed, E., El-Dahshan, K., \& Ghannam, N. (2019). Comparative study for detecting Mobile 456 Application's Anti-Patterns. In the 8th International Conference on Software and Information 457 Engineering (ICSIE 2019) ACM (ISBN:798-1-4503-4503-6618-2) P5:13, Ei Compendex and 458 Scopus, The British University, Cairo, Egypt April 9-12, http://www.icsie.org/

459 Happel, H. J., \& Seedorf, S. (2006, November). Applications of ontologies in software 460 engineering. In Proc. of Workshop on Semantic Web Enabled Software Engineering"(SWESE) 461 on the ISWC, 5-9.

462 Hecht, G., Benomar, O., Rouvoy, R., Moha, N., \& Duchien, L. (2015, November). Tracking the 463 software quality of Android applications along their evolution ( $\mathrm{t}$ ). In 30th IEEE/ACM

464 International Conference on Automated Software Engineering (ASE), 9-13 Nov. 2015; Lincoln, 465 NE, USA. Washington, DC, USA: IEEE, pp. 236-247.

466 Hecht, G., Moha, N., \& Rouvoy, R. (2016, May). An empirical study of the performance impacts 467 of android code smells. In Proceedings of the International Conference on Mobile Software 468 Engineering and Systems, 14-22 May; Austin, Texas. New York, NY, USA: ACM. pp. 59-69.

469 ACM.

470 Hecht, G., Rouvoy, R., Moha, N., \& Duchien, L. (2015, May). Detecting anti-patterns in android 471 apps. In 2nd ACM International Conference on Mobile Software Engineering and Systems 472 (MOBILESoft), 16 - 17 May 2015; Florence, Italy. Piscataway, NJ, USA: IEEE. pp. 148-149.

473 IEEE Standard Glossary of Software Engineering Terminology-Description, (1990).

474 http://ieeexplore.ieee.org/servlet/opac?punumber=2238. Accessed in January 2019.

475 Joorabchi, M. E., Ali, M., \& Mesbah, A. (2015, November). Detecting inconsistencies in multi476 platform mobile apps. In IEEE 26th International Symposium on Software Reliability 477 Engineering (ISSRE), 02 - 05 Nov. 2015; Gaithersbury, MD, USA. IEEE. pp. 450-460.

478 Kappel, G., Kapsammer, E., Kargl, H., Kramler, G., Reiter, T., Retschitzegger, W.,\& Wimmer, 479 M. (2006). Lifting metamodels to ontologies: A step to the semantic integration of modeling 480 languages. In Proceeding of International Conference on Model Driven Engineering Languages 481 and Systems (MODELS), .528-542. Springer. 
482 Khomh, F., Di Penta, M., Guéhéneuc, Y. G., \& Antoniol, G. (2012). An exploratory study of the 483 impact of anti-patterns on class change-and fault-proneness. Empirical Software Engineering, 484 17(3), 243-275.

485 Linares-Vásquez, M., Klock, S., McMillan, C., Sabané, A., Poshyvanyk, D., \& Guéhéneuc, Y. 486 G. (2014, June). Domain matters: bringing further evidence of the relationships among anti487 patterns, application domains, and quality-related metrics in Java mobile apps. In Proceedings of 488 the 22nd International Conference on Program Comprehension02 - 03 June 2014, Hyderabad, 489 India. New York, NY, USA: ACM. pp. 232-243.

490 Maurice, P., Dhombres, F., Blondiaux, E., Friszer, S., Guilbaud, L., Lelong, N., ... \& Jurkovic, 491 D. (2017). Towards ontology-based decision support systems for complex ultrasound diagnosis 492 in obstetrics and gynecology. Journal of gynecology obstetrics and human reproduction, 46(5), $493 \quad 423-429$.

494 Morales, R., Saborido, R., Khomh, F., Chicano, F., \& Antoniol, G. (2016). Anti-patterns and the 495 energy efficiency of Android applications. arXiv preprint arXiv:1610.05711.

496 Obrst, L., Grüninger, M., Baclawski, K., Bennett, M., Brickley, D., Berg-Cross, G., \& Lange, C. 497 (2014). Semantic web and big data meet applied ontology. In Applied Ontology, 9(2), pp. 155498170.

499 Parnas, D. L. (1994, May). Software aging. In Proceedings. Of 16th International Conference on 500 Software Engineering ICSE-16., 16-21 May; Sorrento, Italy. Los Alamitos, CA, USA: IEEE. pp. $501279-287$.

502 Palomba, F., Di Nucci, D., Panichella, A., Zaidman, A., \& De Lucia, A. (2017, February). 503 Lightweight detection of Android-specific code smells: The a Doctor project. In IEEE 24th 504 International Conference on Software Analysis, Evolution and Reengineering (SANER), 20-24 505 Feb. 2017, Klagenfurt, Austria. IEEE. pp. 487-491.

506 Raja, V. (2008). Introduction to reverse engineering. In Raja V., Fernandes K, editors. Reverse 507 Engineering. Springer Series in Advanced Manufacturing. Springer, London, 2008. pp. 1 - 9.

508 Romano, D., Raila, P., Pinzger, M., \& Khomh, F. (2012, October). Analyzing the impact of anti509 patterns on change-proneness using fine-grained source code changes. In 19th Working 510 Conference on Reverse Engineering (WCRE), 15-18 Oct.; Kingston, ON, Canada. Washington, 511 DC, USA: IEEE. pp. 437-446.

512 Song, L., Tang, Z., Li, Z., Gong, X., Chen, X., Fang, D., \& Wang, Z. (2017, December). AppIS: 513 Protect Android Apps Against Runtime Repackaging Attacks. In 2017 IEEE 23rd International 514 Conference on Parallel and Distributed Systems (ICPADS), 25-32. IEEE.

515 Yamashita, A., \& Moonen, L. (2013, May). Exploring the impact of inter-smell relations on 516 software maintainability: An empirical study. In Proceedings of the 2013 International 517 Conference on Software Engineering (pp. 682-691). IEEE Press.

518 Yus, R., \& Pappachan, P. (2015, October). Are Apps Going Semantic? A Systematic Review of 519 Semantic Mobile Applications. In Mobile Deployment of Semantic International Workshop 520 MoDeST@ ISWC, Bethlehem, PA, USA. pp. 2-13.

521 Zhou, X., Wu, K., Cai, H., Lou, S., Zhang, Y., \& Huang, G. (2018). LogPruner: detect, analyze 522 and prune logging calls in Android apps. Science China Information Sciences, 61, pp. 1-3. 


\section{Table $\mathbf{1}$ (on next page)}

The description of the mobile apps under analysis 


\begin{tabular}{|c|c|c|c|}
\hline Mobile Application Name & Size(MB) & Downloads & Description of use \\
\hline Test DPC 4.0.5 & 3.14 & 1.076 .791 & Libraries \& Demo \\
\hline Avast 6.5.3 Security & 20.71 & 1.364 & $\begin{array}{l}\text { Antivirus Engine \& } \\
\text { Mobile Security }\end{array}$ \\
\hline Free-Calls-Messages & 31.59 & 1.537 & Communication \\
\hline Beautiful Gallery 2.3 & 11.31 & 497 & Photography \\
\hline Play Store 9.3.4 & 14.17 & 6.950 & Google Play Store \\
\hline Wall Paper 1.2.166 & 2.29 & 9.730 & Personalization \\
\hline Oasis-Feng/Island 2.5 & 2.34 & 822 & $\begin{array}{l}\text { privacy protection and } \\
\text { parallel running }\end{array}$ \\
\hline Netflix-5-4-0-Build & 18.81 & 22.043 & Entertainment \\
\hline Remainder 1.4 .02 & 9.36 & 3.612 & Remainder \\
\hline Sound-Picker 8.0.0 & 3.9 & 2.142 & Samsung Sound picker \\
\hline Air-Command 2.5.15 & 0.82 & 1.747 & Air command \\
\hline Lifesum-Healthy-Lifestyle & 31.4 & 3.594 & $\begin{array}{l}\text { Diet plan, food diary, } \\
\text { macro calculator, calorie } \\
\text { counter \& healthy recipes }\end{array}$ \\
\hline Background-Defocus 2.2.9 & 3.45 & 2.960 & Photography \\
\hline Gasbuddy-Find-Cheap-Gas & 29.64 & 334 & Travel and Local \\
\hline Soundcloud -Music-Audio.03.03 & 33.2 & 2,066 & Music and Audio \\
\hline Network-Monitor-Mini 1.0.197 & 2.88 & 307 & $\begin{array}{l}\text { Monitor the upload and } \\
\text { download speed per } \\
\text { second }\end{array}$ \\
\hline Casper Android 1.5.6.6 & 18.77 & 383.765 & Messaging app Snapchat \\
\hline Line 8.4.0 & 70.25 & 260 & Communication \\
\hline Diagnosises & 6.96 & 36 & Medical \\
\hline Viber 7.7.0.21 & 38.4 & 1.628 & Communication \\
\hline
\end{tabular}




\begin{tabular}{llll} 
WhatsApp 2.17.235 & 35.81 & 28.978 & Communication \\
Firefox 56.0 & 40.62 & 20.423 & Communication \\
$\begin{array}{l}\text { Blue- Email And Calendar } \\
\text { 1.9.3.21 }\end{array}$ & 43.2 .4 & 203 & Productivity \\
Google Camera 5.1.011.17 & 36.48 & 211.822 & Photography \\
YouTube 13.07 & 24.13 & 23.667 & Video Players \\
True Caller 8.84.12 & 23.09 & 609 & Communication \\
Samsung Gallery 5.4.01 & 17.61 & 10.712 & Photography \\
& & & News and Magazines \\
Twitter 7.48.0 & 35.82 & 694 & Communication \\
Chrome Browser 66.0.3359 & 41.51 & 29.129 & \\
\hline
\end{tabular}




\section{Table 2 (on next page)}

Ten Modelio anti-patterns and their correction way 


\begin{tabular}{ll}
\hline The anti-pattern & The Method of Correction \\
\hline $\begin{array}{l}\text { NameSpaces have the same name. } \\
\text { NameSpace is Leaf and is derived. }\end{array}$ & $\begin{array}{l}\text { Change the name of the conflicting } \\
\text { NameSpaces } \\
\text { Make the NameSpace non-final. }\end{array}$ \\
$\begin{array}{l}\text { NameSpace is Leaf and is abstract. } \\
\text { Meneralization between two }\end{array}$ & $\begin{array}{l}\text { Change the source or the target in order to } \\
\text { link two compatible elements. }\end{array}$ \\
$\begin{array}{l}\text { A public association between two } \\
\text { Classifiers one of them is public and } \\
\text { the other has different visibility. }\end{array}$ & $\begin{array}{l}\text { Change the visibility of the target class to } \\
\text { public. }\end{array}$ \\
$\begin{array}{l}\text { Classifier has several operations with } \\
\text { the same signature. }\end{array}$ & $\begin{array}{l}\text { Rename one of the Operations or change } \\
\text { their parameters. }\end{array}$ \\
$\begin{array}{l}\text { Classifier has attributes with the same } \\
\text { name. }\end{array}$ & $\begin{array}{l}\text { Rename the Classifiers Attributes. } \\
\text { MultiplicityMin must be inferior to } \\
\text { MultiplicityMax. }\end{array}$ \\
$\begin{array}{l}\text { The status of an Attribute is abstract } \\
\text { and class at the same time. }\end{array}$ & $\begin{array}{l}\text { Change the value of the minimum } \\
\text { multiplicity to be less than the maximum } \\
\text { multiplicity. }\end{array}$ \\
\hline
\end{tabular}




\section{Table 3 (on next page)}

OntoUML anti-patterns and the correction way 


\begin{tabular}{ll}
\hline The anti-pattern & The Method of Correction \\
\hline Association Cycle. & Chang the cycle to be closed or open cycle. \\
Binary relation with & $\begin{array}{l}\text { Declare the relation as anti-reflexive, asymmetric and } \\
\text { anti-transitive. }\end{array}$ \\
Overlapping Ends. & $\begin{array}{l}\text { Add domain-specific constraints to refer to which } \\
\text { subtypes of the association end to be an instance of the } \\
\text { other end may be related. }\end{array}$ \\
Imprecise Abstraction. & $\begin{array}{l}\text { Add OCL constraints which guarantee that if there is a } \\
\text { relation between two types and one of them has subtypes, } \\
\text { there must be constraints says that the subtypes are also } \\
\text { in a relation with the other type. }\end{array}$ \\
Relation Composition. & $\begin{array}{l}\text { Add constraints on the relation between the type and the } \\
\text { super-type, declaring that the type is to be either a } \\
\text { specialization, a subset, a redefinition or disjoint with } \\
\text { relation SR }\end{array}$ \\
Relation Specialization
\end{tabular}




\section{Table 4 (on next page)}

Occurrences of the anti-patterns in the mobile apps 


\begin{tabular}{lll}
\hline The Group & $\begin{array}{l}\text { Percentage of occurrences } \\
\text { across models }\end{array}$ & $\begin{array}{l}\text { Total \# of } \\
\text { occurrences }\end{array}$ \\
\hline Anti-patterns in Attributes & $0.713 \%$ & 9 \\
Anti-patterns in & $7.210 \%$ & 91 \\
Namespaces & & 5 \\
$\begin{array}{l}\text { Anti-patterns in } \\
\text { Operations }\end{array}$ & $0.396 \%$ & 554 \\
$\begin{array}{l}\text { Anti-patterns in } \\
\text { Associations }\end{array}$ & $43.898 \%$ & 603 \\
Anti-patterns in the Class & $47.78 \%$ & 1262 \\
Total & & \\
\hline
\end{tabular}




\section{Table 5 (on next page)}

The anti-patterns in each app 


\begin{tabular}{|c|c|c|c|c|c|c|c|c|c|c|c|c|c|c|c|c|c|}
\hline & $\begin{array}{l}\text { Mobile } \\
\text { App }\end{array}$ & 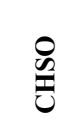 & $\begin{array}{l}\mathbf{Z} \\
\mathbf{n} \\
\mathbf{Z}\end{array}$ & 妾 & $\frac{\sum}{2}$ & 空 & 芯 & $\sum_{\Sigma}^{\sum} \Sigma$ & $\hat{\overline{0}}$ & $\sum_{i}^{u}$ & 气̂ & 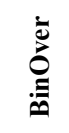 & $\underset{\psi}{U}$ & $\mathscr{\approx}$ & 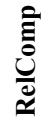 & $\frac{n}{a}$ & है \\
\hline 1 & $\begin{array}{l}\text { Test } \\
\text { DPC 4.0.5 }\end{array}$ & 7 & 2 & 1 & & - & 2 & - & 1 & - & - & 10 & 6 & 1 & - & - & 30 \\
\hline 2 & $\begin{array}{l}\text { Avast } \\
\text { Android } \\
\text { Mobile } \\
\text { Security }\end{array}$ & 149 & 15 & & 2 & - & 58 & 3 & - & 2 & & 6 & - & - & 2 & 3 & 240 \\
\hline 3 & $\begin{array}{l}\text { Free-Calls- } \\
\text { Messages }\end{array}$ & 4 & 1 & 2 & - & 2 & 1 & 1 & - & - & - & 3 & 2 & - & - & - & 16 \\
\hline 4 & $\begin{array}{l}\text { Beautiful } \\
\text { Gallery } 2.1\end{array}$ & 5 & 2 & - & - & - & - & - & 1 & - & - & - & - & - & - & - & 8 \\
\hline 5 & $\begin{array}{l}\text { Play } \\
\text { Store }\end{array}$ & 8 & 1 & 1 & & 1 & 1 & 2 & 3 & 1 & & 6 & 16 & - & 41 & 2 & 82 \\
\hline 6 & $\begin{array}{l}\text { Wall } \\
\text { Paper }\end{array}$ & 1 & 1 & - & - & - & - & 1 & - & - & - & - & - & - & 4 & - & 7 \\
\hline 7 & $\begin{array}{l}\text { Oasis- } \\
\text { Feng/Island }\end{array}$ & 17 & 2 & - & - & - & 4 & - & - & - & - & - & - & - & - & 3 & 26 \\
\hline 8 & $\begin{array}{l}\text { Netflix-5- } \\
\text { 4-0-Build }\end{array}$ & 60 & 7 & - & 2 & - & - & - & - & - & 5 & 5 & - & - & - & - & 79 \\
\hline 9 & Remainder & 11 & 4 & - & - & - & 1 & 2 & - & - & - & 4 & 7 & 5 & - & - & 34 \\
\hline 10 & $\begin{array}{l}\text { Sound- } \\
\text { picker }\end{array}$ & 9 & 1 & - & - & - & - & - & - & - & - & - & - & - & - & 2 & 12 \\
\hline 11 & $\begin{array}{l}\text { Air- } \\
\text { Command }\end{array}$ & 8 & 1 & & & 1 & - & - & - & - & - & - & - & - & - & - & 10 \\
\hline 12 & $\begin{array}{l}\text { Lifesum- } \\
\text { Healthy- } \\
\text { Lifestyle }\end{array}$ & 5 & 1 & - & - & - & - & 1 & - & 4 & - & - & 5 & 1 & 2 & 2 & 21 \\
\hline 13 & $\begin{array}{l}\text { Backgroun } \\
\text { d-Defocus }\end{array}$ & 10 & 4 & - & - & - & 4 & 1 & - & - & - & 10 & - & - & 6 & - & 35 \\
\hline 14 & $\begin{array}{l}\text { Gasbuddy- } \\
\text { Find- } \\
\text { Cheap-Gas }\end{array}$ & 11 & 4 & - & 1 & - & 2 & - & - & 1 & - & - & 7 & 2 & - & 3 & 31 \\
\hline 15 & $\begin{array}{l}\text { Soundclou } \\
\text { d -Music- } \\
\text { Audio }\end{array}$ & 6 & 4 & - & - & - & - & - & 2 & - & - & - & - & 8 & 1 & 2 & 23 \\
\hline 16 & $\begin{array}{l}\text { Network- } \\
\text { Monitor- }\end{array}$ & 7 & 2 & - & - & - & 1 & 2 & - & - & - & - & 3 & - & - & - & 15 \\
\hline
\end{tabular}




\begin{tabular}{|c|c|c|c|c|c|c|c|c|c|c|c|c|c|c|c|c|c|}
\hline \multirow[b]{2}{*}{17} & \multicolumn{17}{|l|}{ Mini } \\
\hline & $\begin{array}{l}\text { Casper } \\
\text { Android }\end{array}$ & 6 & 4 & - & - & - & - & - & 3 & - & - & 20 & - & 6 & - & - & 39 \\
\hline 18 & Line & 15 & 1 & - & - & - & 1 & 1 & - & - & - & - & 6 & - & 2 & 1 & 27 \\
\hline 19 & Diagnoses & 1 & - & - & - & - & - & - & - & - & - & - & 2 & 1 & - & - & 4 \\
\hline 20 & Viber & 42 & 4 & - & - & 1 & 1 & - & - & 1 & - & 9 & - & 7 & 5 & - & 69 \\
\hline 21 & WhatsApp & 5 & 1 & - & - & - & - & 2 & - & - & - & 30 & - & 2 & - & 2 & 42 \\
\hline 22 & Firefox & 40 & 4 & - & - & - & 1 & 1 & 4 & - & - & - & - & 8 & - & 1 & 59 \\
\hline 23 & $\begin{array}{l}\text { Email And } \\
\text { Calendar }\end{array}$ & 15 & 2 & - & - & - & 1 & - & - & - & - & 108 & 2 & - & - & - & 128 \\
\hline 24 & $\begin{array}{l}\text { Google } \\
\text { Camera }\end{array}$ & 9 & 1 & - & - & - & - & - & 1 & - & - & 15 & 8 & - & 1 & 1 & 36 \\
\hline 25 & YouTube & 21 & 4 & - & - & - & 3 & - & - & - & - & 3 & 3 & - & 3 & 2 & 39 \\
\hline 26 & True Caller & 31 & 2 & - & - & - & - & 2 & - & - & - & 17 & 5 & - & 1 & - & 58 \\
\hline 27 & $\begin{array}{l}\text { Samsung } \\
\text { Gallery }\end{array}$ & 12 & - & - & - & - & - & - & 1 & - & - & - & 9 & 3 & - & 1 & 26 \\
\hline 28 & Twitter & 6 & 2 & - & - & - & - & 1 & - & - & - & 15 & 6 & 1 & 1 & - & 32 \\
\hline 29 & $\begin{array}{l}\text { Chrome } \\
\text { Browser }\end{array}$ & 1 & 4 & 1 & - & - & - & - & - & - & - & 9 & 5 & - & 12 & - & 32 \\
\hline \#of & ppearance & 522 & 81 & 5 & 5 & 5 & 81 & 20 & 16 & 9 & 5 & 270 & 92 & 45 & 81 & 25 & 1262 \\
\hline
\end{tabular}




\section{Table 6(on next page)}

The correlation among anti-patterns groups 


\begin{tabular}{ll}
\hline Anti-patterns & Correlation \\
\hline Attributes \& Namespaces & Coefficient(r) \\
Attributes \& Operations & -0.049 \\
Attributes \& Associations & 0.884 \\
Attributes \& Classes & 0.196 \\
Namespaces \& Operations & 0.342 \\
Namespaces \& Associations & -0.060 \\
Namespaces \& Classes & -0.121 \\
Operations \& Associations & 0.010 \\
Operations \& Classes & 0.345 \\
Associations \& Classes & 0.267 \\
\hline
\end{tabular}




\section{Table 7(on next page)}

The correlation among the three tools 


\begin{tabular}{lll}
\hline Systems & $\begin{array}{l}\text { Correlation } \\
\text { Coefficient(r) }\end{array}$ & Specification \\
\hline Modelio \& OntoUml & -0.032 & $\begin{array}{l}\text { There is a reverse correlation } \\
\text { between Modelio and } \\
\text { OntoUml. }\end{array}$ \\
Modelio \& Protégé & 0.966 & $\begin{array}{l}\text { There is a direct correlation } \\
\text { between Modelio and Protégé. }\end{array}$ \\
Protégé \& OntoUML & -0.060 & $\begin{array}{l}\text { There is a reverse correlation } \\
\text { between Protégé and OntoUml } \\
\text { editor. }\end{array}$ \\
\hline
\end{tabular}




\section{Table 8 (on next page)}

Anti-patterns number before and after merging 


\begin{tabular}{lllcl}
\hline Mobile Apps & Viber & $\begin{array}{c}\text { Whats } \\
\text { App }\end{array}$ & $\begin{array}{c}\text { The integrated } \\
\text { app }\end{array}$ & Total \\
\hline (Merging UML designs) & & & \\
\# of detected anti-patterns & 49 & 8 & 58 & 115 \\
in first method using Modelio & & & \\
& & & & \\
(Merging Ontologies) & & & \\
\# of detected anti-patterns & 51 & & \\
in second method using & & & \\
Protégé & & & \\
\hline
\end{tabular}


Figure 1

The proposed method phases

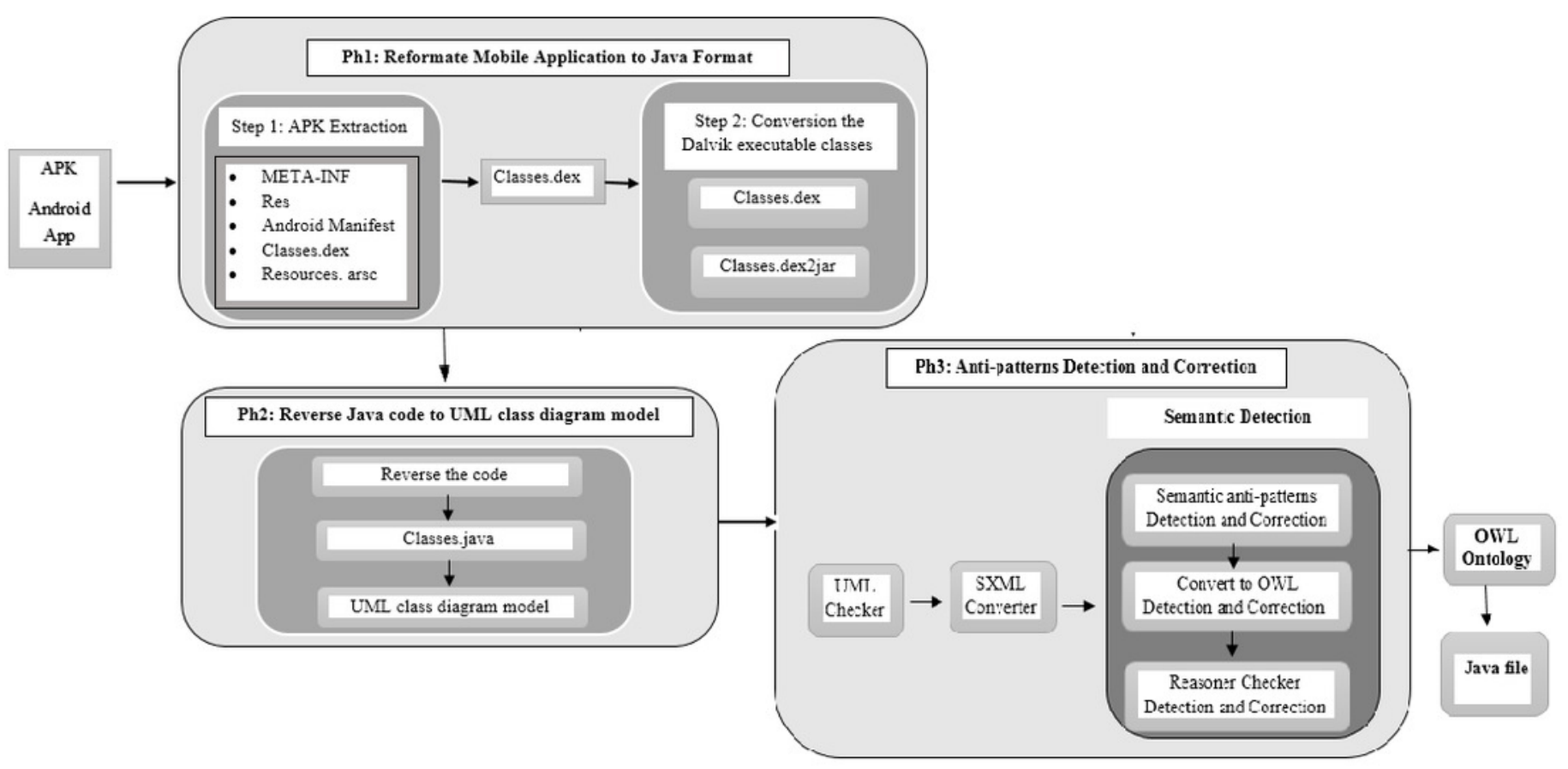


Figure 2

Merging UML class diagrams of the mobile apps

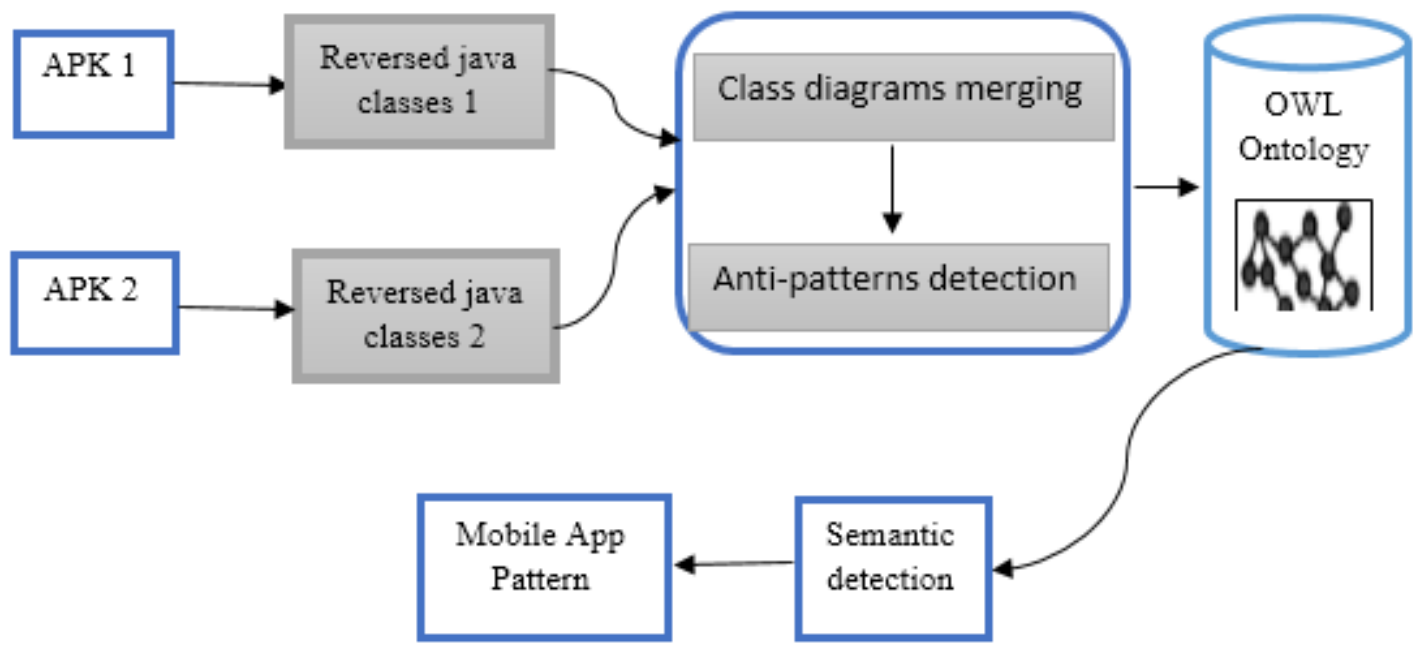


Figure 3

\section{OWL Ontology merging}

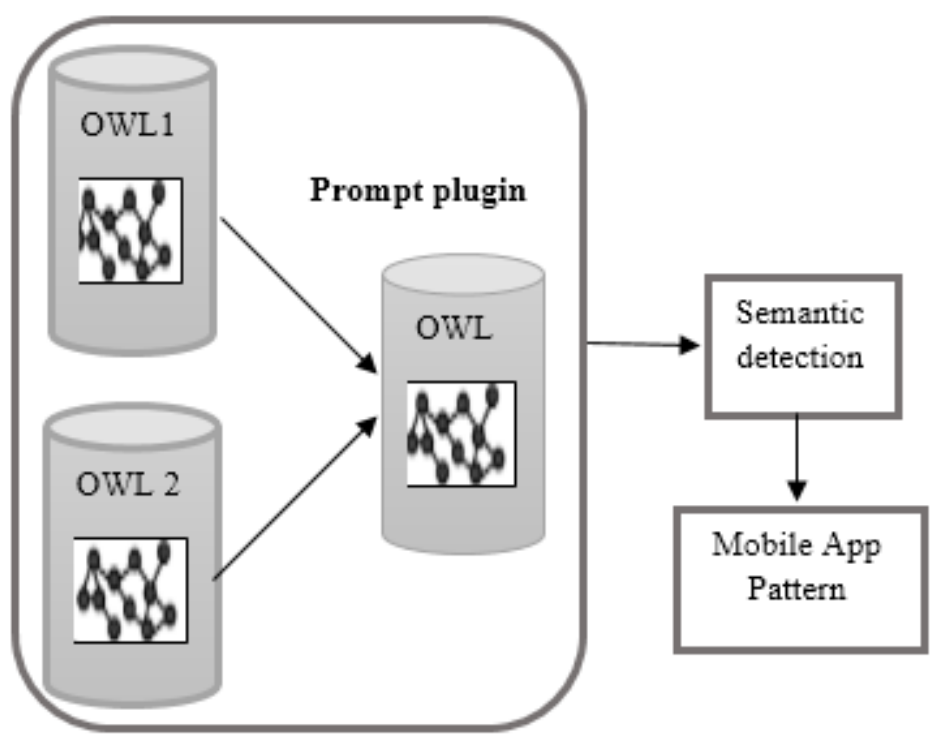




\section{Figure 4}

\section{"Viber and Whatsapp" Ontologies before integration in Protégé}

\begin{tabular}{|c|c|c|}
\hline Name & Arg1 & Arg2 \\
\hline merge & \multicolumn{2}{|c|}{ 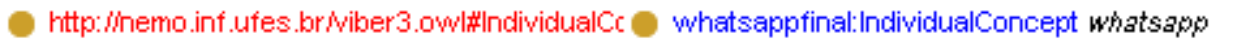 } \\
\hline merge & viber3:TimeSlice viber & whatsappfinal:TimeSlice whatsapp \\
\hline merge & viber3:TemporalExtent viber & whatsapptinal:TemporalExtent whatsapp \\
\hline merge & viber 3 :Object viber & whatsappinal:Object whatsapp \\
\hline merge & viber3:Moment viber & whatsappfinal:Moment whatsapp \\
\hline merge & viber3:Moment viber & whatsappinal:Mode whatsapp \\
\hline merge & viber3:FunctionalComplex viber & whatsappinal:FunctionalComplex whatsapp \\
\hline merge & viber 3 :Collective viber & whatsappinal:Collective whatsapp \\
\hline merge & viber 3 :Collective viber & whatsappinal:CollectionsTS whatsapp \\
\hline merge & viber 3 :Quantity viber & whatsappfinal:Quantity whatsapp \\
\hline merge & viber3:FunctionalComplexTS viber & whatsapprinal:FunctionalComplexTS whatsap \\
\hline merge & viber 3 :CollectiveTS viber & whatsappfinal:CollectiveTS whatsapp \\
\hline merge & viber 3 :CollectiveTS viber & whatsappfinal:CollectionsTS whatsapp \\
\hline merge & viber 3 :QuantityTS viber & whatsappfinal:QuantityTS whatsapp \\
\hline merge & viber 3 Relator viber & whatsappinal:Relator whatsapp \\
\hline merge & viber 3 :RelatorTS viber & whatsapptinal:RelatorTS whatsapp \\
\hline merge & viber3:Mode viber & whatsapptinal:Moment whatsapp \\
\hline merge & viber 3 : Wode viber & whatsapprinal:Mode whatsapp \\
\hline merge & viber3:ModeTS viber & whatsappfinal:ModeTS whatsapp \\
\hline
\end{tabular}




\title{
Figure 5
}

\section{The result slots of the Ontology after integration in Protégé}


Figure 6

\section{The generated UML class diagram of the case study}

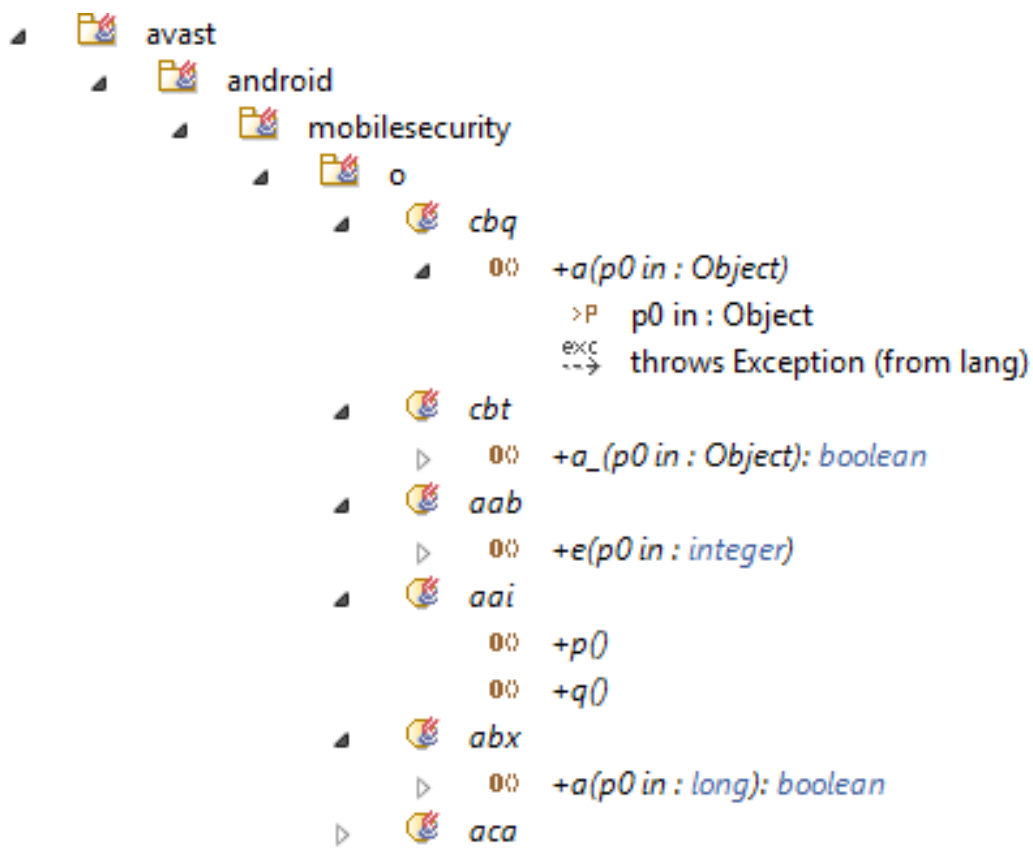




\section{Figure 7}

\section{Modelio anti-patterns}

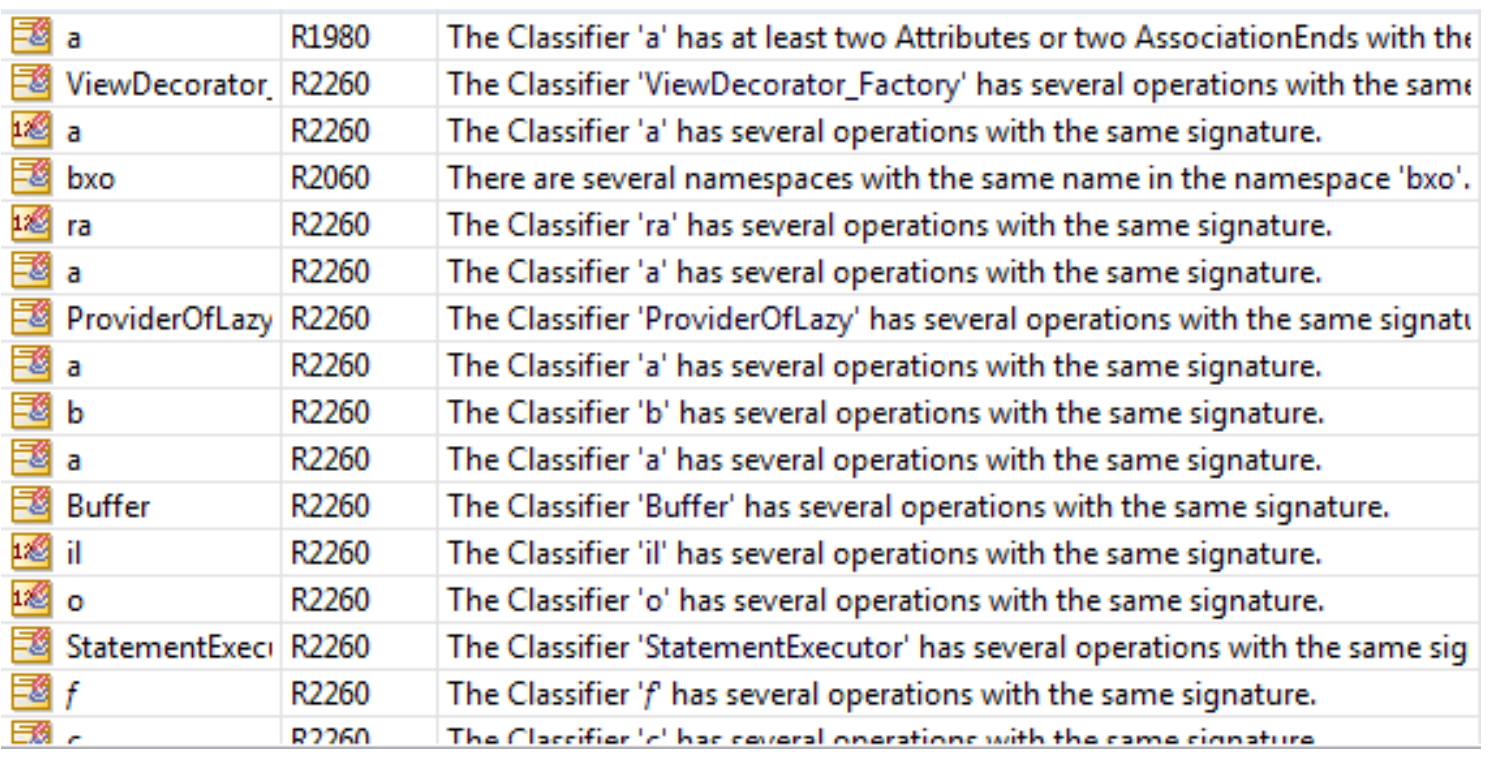




\section{Figure 8}

The anti-pattern " Classifier has several operations with the same signature"

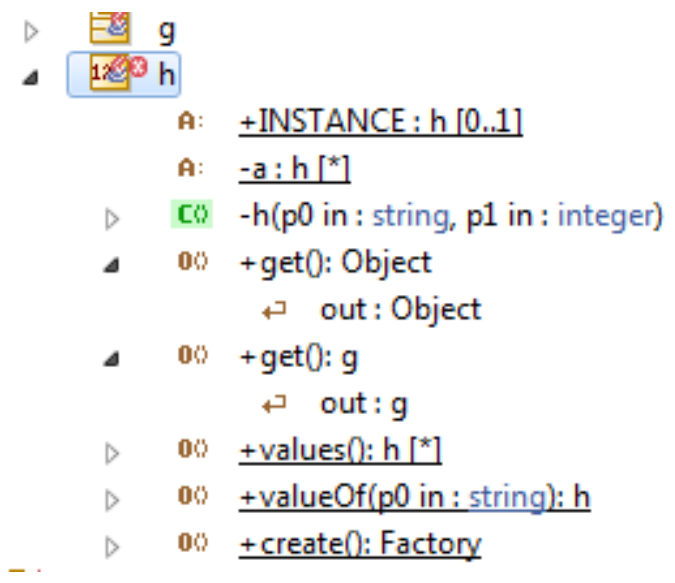




\section{Figure 9}

\section{The inconsistent classes using Reasoner detection}

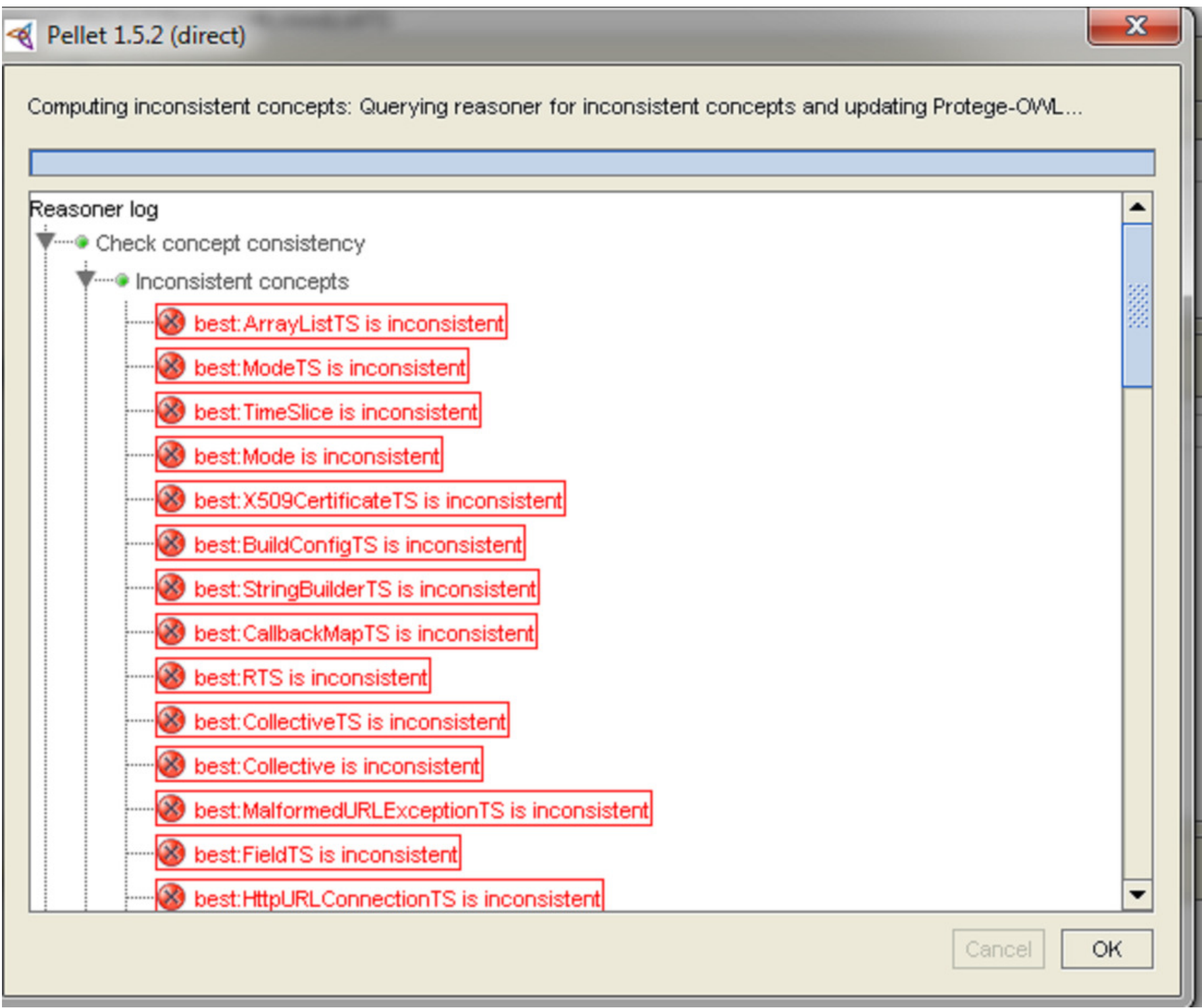




\section{Figure 10}

The occurrences of the detected anti-patterns' groups

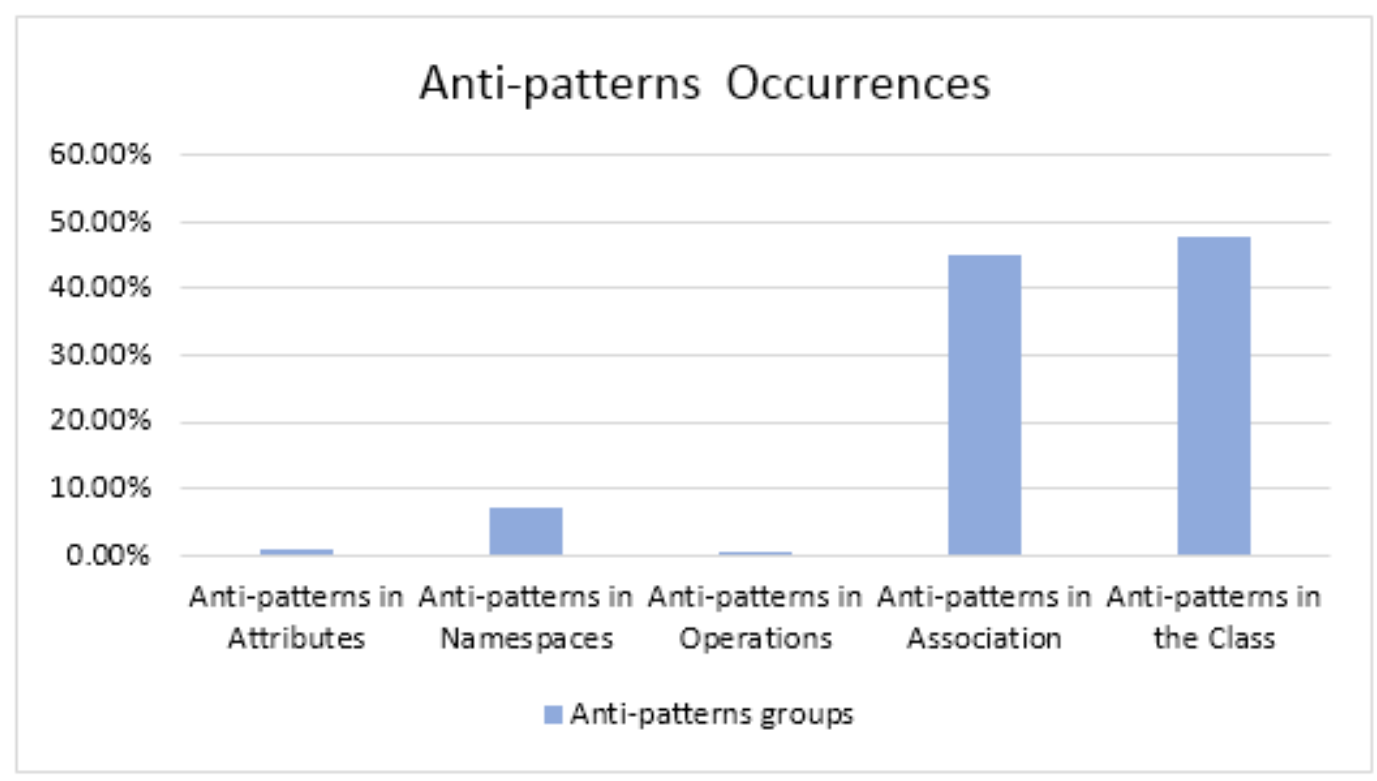


Figure 11

\section{The means of the detected tools}

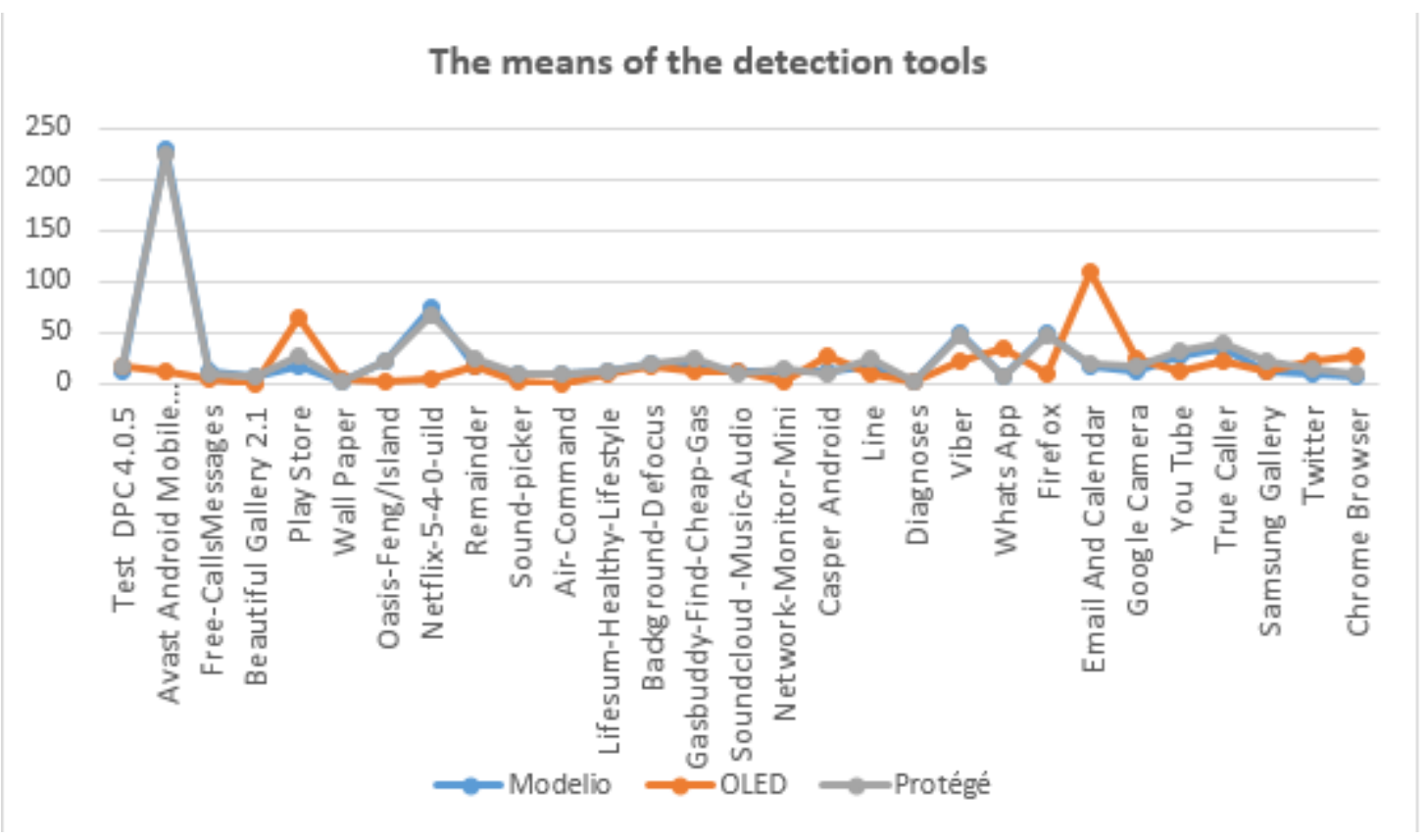

